\title{
Easyspray: software para dimensionamento de sistemas de irrigação por aspersão convencional em regiões moçambicanas
}

\author{
Easyspray: software for solid set sprinkler irrigation \\ systems design in Mozambican regions
}

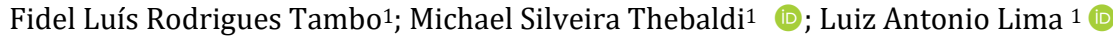 \\ ${ }^{1}$ Universidade Federal de Lavras - UFLA, Lavras, MG, Brasil. E-mails: fideltambo@gmail.com, \\ michael.thebaldi@ufla.br, lalima@ufla.br
}

\begin{abstract}
Como citar: Tambo, F. L. R., Thebaldi, M. S., \& Lima, L. A. (2020). Easyspray: software para dimensionamento de sistemas de irrigação por aspersão convencional em regiões moçambicanas. Revista de Gestão de Água da América Latina, 17, e14.

https://doi.org/10.21168/rega.v17e14

RESUMO: 0 dimensionamento é uma prática indispensável para garantir a viabilidade técnica e econômica de um sistema de irrigação. Devido a diversas variáveis a serem consideradas, ele torna-se cada vez mais difícil de se realizar manualmente. Além disso, a irrigação por aspersão convencional necessita, em alguns casos, da mudança de posição das linhas, e assim o correto dimensionamento é um fator muito importante para sua eficiência hídrica e energética, além de possibilitar análise de cenários de forma a selecionar o tipo de traçado mais econômico. Assim sendo, o principal objetivo deste trabalho foi desenvolver um software para dimensionamento de sistemas de irrigação por aspersão convencional para pequenas propriedades na agricultura familiar em Moçambique. Para isso, utilizou-se o Visual Studio, em que se elaborou uma base de dados com informações climáticas, caraterísticas de solos e dados de principais culturas produzidas em Moçambique. Foram inseridas equações em forma de códigos na linguagem de programação Visual Basic e por meio destas, gerou-se rotinas computacionais para obter uma interface gráfica que permita ao usuário realizar a entrada de dados e observar os resultados obtidos. 0 software apresentou uma interface gráfica e intuitiva, além de ser capaz de ser instalado e funcionar eficientemente, em computadores que usam Windows, sem depender de outros programas auxiliares.
\end{abstract}

Palavras-chave: Hidráulica Agrícola; Gestão da Água na Irrigação; Sistemas de Irrigação para Agricultura Familiar.

ABSTRACT: Correct hydraulic design is necessary to guarantee technical and economical viability of an irrigation system. Due to several variables to be considered, it becomes increasingly difficult to be carried out manually. Furthermore, due to the fact that solid set sprinkler irrigation requires, in some cases, a change in pipe positions, the correct design is very important to obtain water and energy efficiency; in addition it enables analysis of different scenarios, for better selecting an adequate layout. Therefore, the main objective of this work was to develop a software to design solid set sprinkler irrigation systems for small properties and family farmers in Mozambique. For this, Visual Studio was used, in which a database was prepared with climatic information, soil characteristics and data of the main crops produced in Mozambique. Coded equations were inserted into the Visual Basic programming language and through these, computational routines were generated to obtain a graphical interface that allows the user to perform data entry and observe the results obtained. The software presented a graphic and intuitive interface, besides being able to be installed and to work efficiently, in computers that use Windows, without depending on other auxiliary programs.

Keywords: Agricultural Hydraulics; Irrigation Water Management; Irrigation Systems for Family Agriculture.

\section{INTRODUÇÃO}

A irrigação é uma técnica que consiste em fornecer água às culturas agrícolas, com objetivo de minimizar problemas como a falta e a má distribuição das chuvas. Porém, é uma tecnologia que está associada à utilização intensiva de insumos e requer elevados investimentos, sendo necessário um criterioso dimensionamento dos componentes (Geisenhoff et al., 2015; Pereira et al., 2016).

Recebido: Abril 14, 2020. Revisado: Junho 19, 2020. Aceito: Agosto 14, 2020. 
Um sistema de irrigação quando mal dimensionado, pode se tornar inviável em termos técnicos e econômicos; por isso, um bom dimensionamento de um sistema de irrigação é de fundamental importância para economia de água e energia elétrica (Miranda et al., 2018). Além disso, o sistema de irrigação deve ser projetado visando garantir que este opere com a sua máxima eficiência hídrica e energética, para que se possa garantir que ele seja viável (Bertossi et al., 2013; Locatelli et al., 2014).

De acordo com o Ministério da Agricultura de Moçambique - MASA (Moçambique, 2017), o potencial de área irrigável em Moçambique é de aproximadamente 3.000.000 ha, porém, apenas 120.000 ha são irrigados atualmente, o que correspondente a 4\% da área total. Além disso, 70\% dessa área é irrigada utilizando-se o método de irrigação superficial. Com efeito, o MASA (Moçambique, 2017) afirma que o fato de maior parte das áreas destinadas à produção agrícola em Moçambique serem de sequeiro, tem sido um dos principais entraves na produção e ganho de produtividade agrícola, principalmente para o setor familiar.

Além disso, sabe-se que dentre os diversos métodos de irrigação existentes, a irrigação superficial é a que apresenta a menor eficiência de uso de água (Lopes et al., 2017). Uma das razões que levam à fraca expansão e fracasso de sistemas de irrigação de pequenos agricultores do setor familiar, é a pequena disponibilidade de técnicos, as falhas técnicas na elaboração e implementação dos projetos além da indisponibilidade de rede elétrica nas áreas rurais (Moçambique, 2017).

Portanto, o uso de irrigação por aspersão convencional em Moçambique pode ser uma alternativa eficiente para reduzir o uso de sistemas de irrigação que empregam o método de irrigação por superfície. Porém, por esse sistema necessitar de tubos, aspersores, motobombas e movimento das linhas laterais, para o tipo semifixo, o seu dimensionamento pode apresentar graves falhas se elaborado manualmente (Pinto et al., 2015), sendo que o correto dimensionamento é fundamental para garantir uma boa eficiência de um sistema de irrigação (Campêlo et al., 2014). Além disso, em áreas com deficiência de técnicos capacitados os softwares são mais importantes na minimização de tempo gasto no trabalho e além da redução de erros pois possibilitam a análise de cenários mais rapidamente, de forma a se determinar qual tipo de traçado é mais econômico ou eficiente no que se propõe (Geisenhoff et al., 2018; Andrini et al. 2019).

Desta forma, o principal objetivo com este trabalho foi desenvolver um software para dimensionamento de sistema de irrigação por aspersão convencional semifixo para pequenos agricultores do setor familiar, considerando o bombeamento de água realizado por meio de energia elétrica ou à combustão de óleo Diesel. Esta iniciativa busca facilitar a elaboração de projetos de irrigação, permitir o uso eficiente dos recursos hídricos, reduzir os custos de operação nos sistemas de irrigação e tornar a irrigação acessível a regiões rurais.

A escolha pelo uso da irrigação por aspersão convencional deve-se ao fato deste sistema de irrigação ser o mais econômico no uso da água, em comparação com os sistemas de irrigação superficial (Frigo et al., 2016; Rodrigues et al., 2019). Este sistema também, apresenta menor necessidade de manutenção e possibilita o uso de águas de menor qualidade, quando comparados com irrigação localizada (Rodrigues et al., 2013).

\section{MATERIAIS E MÉTODOS}

Os códigos de programação foram desenvolvidos em Visual Studio 2017, para aplicação em Windows Forms (.NET Framework), na linguagem de programação Visual Basic.

Para garantir que o software desenvolvido tenha aplicação em Moçambique, foram considerados os valores locais de evapotranspiração de referência, caraterísticas das principais culturas produzidas no país, como o coeficiente de cultura (Kc), profundidade de sistema radicular (z) e fator de disponibilidade (f) e dados dos solos moçambicanos (umidades na capacidade de campo e no ponto de murcha permanente). Os dados climáticos, referentes à evapotranspiração de referência de todas as localidades moçambicanas foram obtidos e calculados com o auxílio de softwares disponibilizados pela FAO (Food and Agriculture Organization of the United Nations, 2006), sendo o CLIMWAT 2.0 e CROPWAT 8.0, que estão disponíveis gratuitamente on-line. Os dados das principais cultura produzidas em Moçambique, foram obtidos recorrendo-se ao manual 56 da FAO (Food and Agriculture Organization of the United Nations, 1990).

Para facilitar a elaboração e utilização do software, ele foi dividido em três etapas, que são apresentadas como abas ao usuário. Às abas foram atribuídos os seguintes nomes: "Projeto agronômico"; "Linha lateral e principal" e "Estação elevatória".

$\mathrm{Na}$ aba "Projeto agronômico", foram geradas rotinas computacionais, para que o software, de forma automática, pudesse apresentar o mapa de Moçambique com todas as localidades e cidades 
disponíveis identificadas. Ao ser selecionada uma das localidades, o software apresentará os seus dados climáticos.

Nesta aba, foram inseridas também todas as equações em forma de código de programação necessárias para o cálculo do projeto agronômico de um sistema de irrigação por aspersão semifixo. É nessa aba onde calcula-se a disponibilidade total e real de água, lâmina liquida de irrigação, lâmina bruta de irrigação, turno de rega, período de irrigação, tempo de irrigação, quantidade de posições a serem irrigadas por dia e número total de linhas laterais. É também disponibilizada uma saída visual, em janela específica, dos resultados referentes a esses cálculos. Desta forma, para o software calcular a água disponível real no solo, utilizou-se Equação 1, apresentada por Carvalho \& Oliveira (2012), essa equação foi inserida em forma de código de programação.

$\mathrm{ADR}=\left(\theta_{\mathrm{CC}}-\theta_{\mathrm{PMP}}\right) \cdot \mathrm{z} \cdot \mathrm{f}$

Em que: ADR é água disponível real (mm); $\theta_{\mathrm{CC}}$ é a umidade na capacidade de campo $\left(\mathrm{cm}^{3} \mathrm{~cm}^{-3}\right) ; \theta_{\mathrm{PMP}}$ é a umidade no ponto de murcha permanente $\left(\mathrm{cm}^{3} \mathrm{~cm}^{-3}\right)$; e $\mathrm{z}$ é a profundidade da raiz $(\mathrm{mm})$; e f é o fator de disponibilidade de água no solo (decimal). Foi criado um banco de dados contendo informações das principais culturas produzidas em Moçambique, onde os valores de fator de disponibilidade de água (Food and Agriculture Organization of the United Nations, 1990) para essas culturas foram inseridos, de forma a serem apresentados ao usuário do software de forma automática.

Para o cálculo do turno de rega foi utilizada Equação 2, apresentada por Mantovani et al. (2009).

$\mathrm{TR}=\frac{\mathrm{ADR}}{\mathrm{ETc}}$

Em que: TR é o turno de rega (dias); ADR é água disponível real (mm); e ETc é a evapotranspiração da cultura (mm.dia-1).

Depois de se obter o turno de rega, o software calcula a lâmina líquida de irrigação por meio da Equação 3, e a lâmina bruta de irrigação pela Equação 4 (Bernardo et al., 2008).

$\mathrm{LLI}=\mathrm{ETc} . \mathrm{TR}$

$\mathrm{LBI}=\frac{\mathrm{LLI}}{\mathrm{Ef}}$

Em que: LLI é a lâmina líquida de irrigação (mm); LBI é a lâmina bruta de irrigação (mm); e Ef é a eficiência de aplicação de água do sistema de irrigação por aspersão convencional (decimal). Apesar de diversos autores como Frigo et al. (2016) e Rodrigues et al. (2019) citarem que, geralmente, em sistemas de aspersão convencional a eficiência de aplicação de água está entre 80 a 90\%, nesse software introduziu-se uma caixa de texto onde usuário pode digitar o valor de eficiência que pretende considerar para o seu projeto. A recomendação é, entretanto, de 80 a $90 \%$.

Para a obtenção do tempo de irrigação foi inserida a Equação 5 (Bernardo et al., 2008).

$\mathrm{T}_{\mathrm{i}}=\frac{\mathrm{LBI}}{\mathrm{Ia}}$

Já para estimar o número de linhas laterais (NLlat) foi inserida a Equação 6, apresentada pelos autores supracitados, em que: Ti é o tempo de irrigação de um evento (h); LBI é a lâmina bruta de irrigação (mm); e Ia é a intensidade de aplicação do aspersor (mm.h-1).

$\mathrm{NL}_{\text {lat }}=\frac{\text { NPILD }}{\text { NPID }}$

NPILD é o número de posições irrigadas por lateral por dia (adimensional); e NPID é número total de posições a serem irrigadas por dia (adimensional).

Para calcular os valores de NPILD e NPID, foram utilizadas as Equações 7 e 8 respectivamente (Lopes et al., 2017). Assumiu-se para isso a probabilidade de ocorrência da necessidade de um dia para reparos no sistema, diminuindo uma unidade no turno de rega. 
$\mathrm{NPILD}=\frac{\mathrm{J}}{\mathrm{T}_{\mathrm{i}}+\mathrm{T}_{\mathrm{m}}}$

J é a jornada de trabalho (h); $T_{i}$ é o tempo de duração de um evento de irrigação (h); e $T_{m}$ é o tempo de mudança de posições da linha lateral (h).

$\mathrm{NPID}=\frac{\mathrm{NTP}}{\mathrm{TR}-1}$

NTP é o número total de posição na linha principal (adimensional); e TR é o turno de rega (dias).

Para estimar o número total de posições na linha principal (NTP) utilizou-se a Equação 9, apresentada por diversos autores como Mantovani et al. (2009). Nesta, o valor de NTP é arredondado para um número inteiro abaixo do encontrado.

$\mathrm{NTP}=\frac{\mathrm{L}_{\mathrm{p}}}{\mathrm{E}_{\text {lat }}}$

Em que: Lp. é o comprimento da área (m) e Elat é o espaçamento entre linhas laterais (m).

$\mathrm{Na}$ aba "Linha lateral e principal", introduziu-se equações na forma de códigos de programação para que o software possa efetuar o dimensionamento da linha lateral e da linha principal de um sistema de irrigação por aspersão convencional. Para o dimensionamento da linha lateral calcula-se o número de aspersores na linha lateral, vazão da entrada de linha lateral, perda de carga máxima permitida, diâmetro da linha lateral, perda de carga real e pressão no início da linha lateral. Já no dimensionamento da linha principal calcula-se a vazão na linha principal, o diâmetro da linha principal e perda de carga.

Para a estimativa do número de aspersores por cada linha lateral inseriu-se a Equação 10, na forma de código de programação (Lopes; Lima; Oliveira, 2017).

$$
\mathrm{NA}_{\text {lat }}=\frac{\mathrm{L}_{\mathrm{L}}}{\mathrm{E}_{\text {asp }}}
$$

Em que: $\mathrm{NA}_{\text {lat }}$ corresponde ao número de aspersores por cada linha lateral (adimensional); $\mathrm{L}_{\mathrm{L}}$ é o comprimento da linha lateral (m); e Easp é o espaçamento entre aspersores (m).

Para calcular a vazão da linha lateral, inseriu-se a Equação 11, que leva em consideração o número de aspersores na linha lateral e a vazão do aspersor (Lopes et al., 2017).

$\mathrm{Q}_{\mathrm{L}}=\mathrm{q} \cdot \mathrm{NA}_{\text {lat }}$

Em que: $\mathrm{Q}_{\mathrm{L}}$ é a vazão da linha lateral $\left(\mathrm{m}^{3} \mathrm{~h}^{-1}\right)$; $\mathrm{q}$ é a vazão do aspersor $\left(\mathrm{m}^{3} \mathrm{~h}^{-1}\right)$; e $\mathrm{NA}_{\text {lat }}$ corresponde ao número de aspersores por cada linha lateral (adimensional).

No cálculo da perda de carga máxima permitida, adotou-se o critério que considera que esta não deve ultrapassar 20\% da pressão de serviço do aspersor, inserindo-se à Equação 12, em que o desnível da linha lateral é calculado pela diferença de cotas no início e no final da linha (Mantovani et al., 2009); desse forma, foram inseridas duas caixas de texto que permitem ao usuário digitar as cotas no início e no fim da lateral.

$\mathrm{hf}_{\max }=0,2 \cdot \mathrm{PS} \pm \Delta \mathrm{Z}$

Em que: $\mathrm{hf}_{\max }$ corresponde a perda de carga máxima permitida (mca); PS é a pressão de serviço do aspersor (mca); e $\Delta Z$ é o desnível do terreno no sentido da linha lateral (m).

Para o software estimar o diâmetro da linha lateral recorreu-se a Equação 13, conhecida como equação de Hazen-Williams em que é possível considerar o emprego de diversos tipos de material (plásticos, aço galvanizado, ferro fundido, ferro corrugado), a fim de permitir que o usuário selecione o material que pretende utilizar no sistema de irrigação (Carvalho, 2014). Além disso, considerou-se a perda da carga máxima permitida na linha lateral de irrigação $\left(\mathrm{hf}_{\max }\right)$, e o fator de correção de Christiansen de perda de carga devido a múltiplas saídas modificado por Scallopi (Mantovani et al., 2009). 
$\mathrm{D}_{\mathrm{L}}=\left[\frac{10,64 \cdot \mathrm{L}_{\mathrm{L}} \cdot\left(\frac{\mathrm{Q}_{\mathrm{L}}}{3600}\right)^{1,852}}{\mathrm{C}^{1,852} \cdot(0,2 \cdot \mathrm{PS} \pm \Delta \mathrm{Z})} \cdot \mathrm{Fa}\right]^{1 / 4,87}$

Em que: $\mathrm{D}_{\mathrm{L}}$ é o diâmetro da linha lateral (m); hf max é a perda de carga máxima permitida na linha lateral (mca); Fa é o fator de correção de Christiansen de perda de carga modificado por Scaloppi (decimal); e C é o coeficiente de atrito, adimensional, de Hazen-Williams do material (Carvalho, 2014).

0 fator de correção de Christiansen de perda de carga modificado por Scaloppi, utilizando à Equação 14, apresentada por Miranda et al. (2018).

$$
\mathrm{Fa}=\frac{\left(\mathrm{NA}_{\text {lat }} \cdot \mathrm{F}\right)+\mathrm{X}-1}{\mathrm{NA}_{\text {lat }}+\mathrm{X}-1}
$$

Em que: $\mathrm{NA}_{\text {lat }}$ é o número de aspersores na linha lateral (adimensional); X é a razão entre a distância do primeiro aspersor e o espaçamento regular entre os aspersores (decimal); e F é o fator de correção de perda de carga de Christiansen para múltiplas saídas (decimal).

Para obtenção do fator de correção de perda de carga de Christiansen, utilizou-se à Equação 15 (Miranda et al., 2018).

$\mathrm{F}=\frac{1}{\mathrm{~m}+1}+\frac{1}{2 \cdot \mathrm{NA}_{\mathrm{lat}}}+\frac{\sqrt{\mathrm{m}-1}}{6 \cdot\left(\mathrm{NA}_{\mathrm{lat}}\right)^{2}}$

Em que: m é o expoente da vazão na equação de perda de carga (adimensional); nesse caso o software considera $(m=1,85)$. Para o cálculo da perda de carga real da linha lateral, foi utilizado equação de Hazen-Williams (Equação 16), apresentada por Carvalho (2014).

$\mathrm{hf}_{\text {lat }}=\frac{10,64 \cdot \mathrm{L}_{\mathrm{L}} \cdot\left(\frac{\mathrm{Q}_{\mathrm{L}}}{3600}\right)^{1,852}}{\mathrm{C}^{1,852} \cdot \mathrm{D}_{\mathrm{L}}^{4,87}} \cdot \mathrm{Fa}$

Em que: hflat: é a perda de carga na linha lateral (mca); e $\mathrm{D}_{\mathrm{L}}$ é o diâmetro da linha lateral (m). Para a determinação da pressão no início da linha lateral considerou-se que $75 \%$ da perda de carga da linha lateral ocorre até a posição relativa ao valor médio de pressão (Pressão de serviço), inserindo-se à Equação 17 (Bernardo et al., 2008).

Pin $=\mathrm{PS}+\mathrm{Aa}+0,75 \cdot \mathrm{hf}_{\text {lat }} \pm 0,5 . \Delta \mathrm{Z}$

Em que: Pin é a pressão no início da linha lateral (mca); $\mathrm{hf}_{\text {lat }}$ é a perda de carga na linha lateral (mca); Aa é altura de elevação do aspersor (m); e $\Delta \mathrm{Z}$ é desnível da linha lateral (m).

Para a estimativa do diâmetro da linha principal, foi utilizado equação de continuidade, estabelecendo-se uma velocidade máxima de $1,5 \mathrm{~m} \mathrm{~s}^{-1}$, que de acordo com Perroni et al. (2015) é um valor dentro da faixa econômica de dimensionamento. Para cálculo da perda de carga na linha principal, foi utilizada a equação de Hazen-Williams, apresentada por Azevedo Neto (2015) (Equação 18).

$\mathrm{hf}_{\mathrm{LP}}=\frac{10,64 \cdot \mathrm{L}_{\mathrm{P}} \cdot\left(\mathrm{Q}_{\mathrm{p}} / 3600\right)^{1,852}}{\mathrm{C}^{1,852} \cdot \mathrm{D}_{\mathrm{P}}^{4,87}}$

Em que: $L_{P}$ é o Comprimento da linha principal $(\mathrm{m}) ; \mathrm{D}_{\mathrm{P}}$ é o diâmetro da linha principal (m); e $\mathrm{Q}_{\mathrm{p}}$ é vazão do projeto $\left(\mathrm{m}^{3} \mathrm{~h}^{-1}\right)$. Para cálculo da vazão do projeto de irrigação é empregada a Equação 19, que leva em consideração a vazão na linha lateral $\left(\mathrm{Q}_{\mathrm{L}}\right)$ e número de linha laterais (NLlat), conforma citado por Pereira et al. (2016).

$\mathrm{Q}_{\mathrm{p}}=\mathrm{Q}_{\mathrm{L}} \cdot \mathrm{NL}_{\text {lat }}$ 
Na aba "Estação elevatória", é realizado o dimensionamento da adutora e da linha de sucção e motobomba.

Para o software calcular as perdas de carga na adutora e linha de sucção foi inserida a Equação 18, porém, considerando os comprimentos e diâmetros correspondentes à adutora e sucção. Já as perdas de carga localizadas ( $\mathrm{hf}_{\mathrm{loc}}$ ) foram adotadas como sendo $5 \%$ do somatório de todas as perdas de carga que ocorrem no sistema de irrigação (Silva \& Klar, 2010). Para calcular a altura manométrica inseriu-se a Equação 20, que considera a pressão no início da linha lateral (Equação 17), a perda de carga total contínua da linha principal, adutora e sucção $\left(\mathrm{hf}_{\text {total }}\right)$, as perdas de carga localizadas $\left(\mathrm{hf}_{\text {loc }}\right)$, e altura geométrica total $\left(\mathrm{Hg}_{\mathrm{T}}\right)$ que contempla os desníveis das linhas principal, adutora e de sucção (Bernardo et al., 2008).

$\mathrm{Hman}=\mathrm{Pin}+\mathrm{hf}_{\text {total }}+\mathrm{Hg}_{\mathrm{T}}+\mathrm{hf}_{\text {loc }}$

Em que: $\mathrm{Hg}_{\mathrm{T}}$ é a altura geométrica total ( $\left.\mathrm{m}\right)$; e $\mathrm{hf}_{\mathrm{loc}}$ é a perda de carga localizada (mca)

Já para o cálculo da potência a ser absorvida pelo conjunto motor e bomba utilizou-se a Equação 21, apresentada por Azevedo Neto (2015), sendo a escolha e entrada dos rendimentos do motor e da bomba deixados a critério do usuário, por meio da disponibilização de duas caixas de texto.

$\mathrm{P}_{\mathrm{abs}}=\frac{\mathrm{Q}_{\mathrm{p}} \cdot \mathrm{Hman}}{270 \cdot \eta_{\mathrm{B}} \cdot \eta_{\mathrm{M}}}$

Em que: $P_{a b s}$ é a potência absorvida (c.v.); $Q_{p}$ é a vazão do projeto $\left(\mathrm{m}^{3} \mathrm{~h}^{-1}\right)$; Hman é a altura manométrica total (mca); $\eta_{\mathrm{B}}$ é o rendimento da bomba, adimensional (decimal); e $\eta_{\mathrm{M}}$ é o rendimento do motor, adimensional (decimal).

Como diversas regiões rurais de Moçambique não possuem rede elétrica convencional, foi implementada no software a capacidade de dimensionamento de motobombas que funcionam por meio de combustível fóssil (óleo Diesel). Nesse caso, são calculados o consumo específico e quantidade de óleo Diesel consumido durante um evento de irrigação. Para cálculo do consumo específico da motobomba à Diesel, foi inserida a Equação 22 (Carvalho, 2014).

$\mathrm{Ce}=\left(0,03054+\frac{0,2445}{\mathrm{P}}\right)^{0,5}$

Em que: Ce é o consumo específico de óleo Diesel $\left(\mathrm{L} \mathrm{cV}^{-1} \mathrm{~h}^{-1}\right)$; e $\mathrm{P}$ é a potência do motor na rotação de melhor desempenho (cv), normalmente a $1800 \mathrm{rpm}$. Finalmente, o volume de óleo Diesel consumido para um evento de irrigação é determinado pelo uso da Equação 23 (Carvalho, 2014).

$\mathrm{Vd}=\mathrm{Ce} . \mathrm{P} . \mathrm{J}$

Em que: Vd é o volume de óleo Diesel consumido (L); J é a Jornada de trabalho (h).

Para testar e validar o funcionamento do software, foi dimensionado um sistema de irrigação de uma associação de produtores rurais no distrito de Chimoio em Moçambique, e os resultados obtidos foram comparados com os do dimensionamento realizado manualmente. A área para o projeto era de 10 ha, especificando-se uma jornada de trabalho de 12 horas. A cultura definida foi o feijão com $60 \mathrm{~cm}$ de profundidade efetiva do sistema radicular, 1,05 de coeficiente de cultura, 0,45 de fator de disponibilidade de água no solo. 0 solo local é argiloso com 10,5 mm/h de velocidade de infiltração básica, 43,75\% de umidade na capacidade de campo e 21,25\% de umidade no ponto de murcha permanente, ambas com base em volume. De acordo com a velocidade de infiltração da água no solo, foram adotados aspersores com 3,2 $\mathrm{m}^{3} \mathrm{~h}^{-1}$ de vazão, espaçamento máximo de 24 x $24 \mathrm{~m}$ e 30 mca de pressão de serviço, que gera uma intensidade de aplicação de água de $6 \mathrm{~mm} \mathrm{~h}^{-1}$.

\section{RESULTADOS E DISCUSSÃO}

A interface gráfica inicial do software após o desenvolvimento das rotinas computacionais está apresentada na Figura 1. Na parte superior apresentam-se as quatro abas, nomeadamente: "Projeto agronômico"; "Linha lateral e principal" e "Estação elevatória", que, por meio destas, o usuário pode efetuar o dimensionamento do sistema de irrigação. Abaixo da aba "Projeto agronômico", apresenta-se 
o mapa de Moçambique com as localidades devidamente identificadas, onde o usuário pode selecionar a localidade em que pretende instalar o sistema de irrigação.

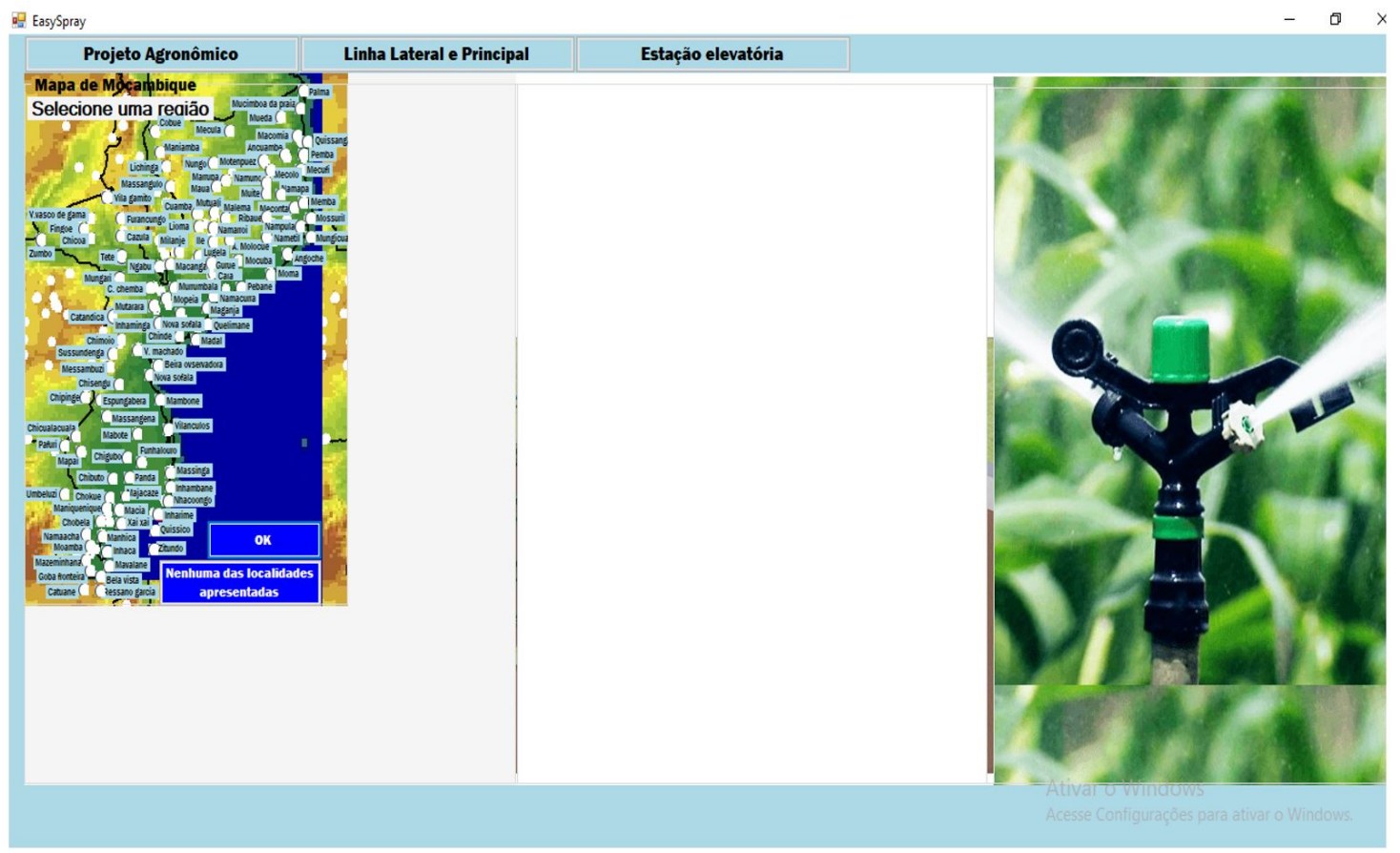

Figura 1 - Apresentação da interface gráfica inicial do software

Quando o usuário seleciona o comando "Projeto agronômico", este permite-lhe iniciar o dimensionamento do sistema de irrigação; ao ser apresentada a caixa "dados gerais", o software solicita a digitação dos dados relacionados à cultura, solo e dados do aspersor. Para dados de cultura e solo, o software apresenta um comando de ajuda, que ao ser selecionado, é mostrada uma caixa onde o usuário pode selecionar uma cultura e um tipo de solo de acordo com o que se tem localmente. Já para dados do aspersor, o usuário deverá obtê-los por meio de catálogos de fabricantes disponíveis.

Após esta entrada de dados, deve-se pressionar o comando "calcular"; assim, o software apresenta os resultados do projeto agronômico de um sistema de irrigação, com os resultados referentes à disponibilidade total e real de água no solo, lâmina bruta de irrigação, turno de rega, tempo de irrigação, período de irrigação, número de posição a serem irrigadas por dia e número e linhas laterais.

Abaixo da caixa de digitação de dados, apresenta-se o nome da cultura selecionada e, ao lado do comando "calcular", o software apresenta opção "voltar". O uso deste comando leva o usuário à tela anterior, que neste caso será a interface gráfica inicial do software. Quando se compara o Easyspray com software IRRIGAR, desenvolvido por Geisenhoff et al. (2018), o primeiro proporciona maior facilidade ao usuário para a aquisição de dados a serem utilizados no projeto agronômico, pois já há uma biblioteca com os dados climáticos, da cultura e de solo que facilitam a elaboração de projetos.

Em contrapartida, uma das vantagens do software IRRIGAR (Geisenhoff et al., 2018) é que, além do projeto agronômico e dimensionamento hidráulico do sistema de irrigação, este realiza também cálculo do custo dos materiais necessários para implantação do sistema, fato que auxilia o projetista na tomada de decisão por meio de quesitos econômicos. Ademais, o software desenvolvido por Geisenhoff et al. (2018) pode ser utilizado para dimensionamentos de sistemas, porém, com interface gráfica mais complexa, enquanto o Easyspray é aplicável apenas a Moçambique, contudo, com interface gráfica simplificada. A interface gráfica do software Easyspray, depois de se efetuar o uso de comando "Projeto agronômico", pode ser vista na Figura 2. Nesta, pode-se verificar todos os comandos mencionados anteriormente.

O comando "Linha lateral e principal" permite efetuar o dimensionamento da linha lateral e linha principal do sistema de irrigação. Quando o usuário seleciona este comando, o software abre uma caixa em que se deve digitar os seguintes dados: material de fabricação dos tubos, coeficientes de HazenWilliams, distância do primeiro aspersor, cotas no início e no final da linha lateral, altura de elevação 
do aspersor e diâmetro interno da linha lateral, que são necessários no dimensionamento da linha lateral. 0 coeficiente de Hazen-Williams é apresentado automaticamente, assim que é selecionado o material de fabricação da tubulação. Com a seleção do comando "calcular", os cálculos são realizados e os resultados apresentados, sendo: número de aspersor na linha lateral, vazão na entrada da linha lateral, comprimento real da linha lateral, perda de carga máxima na linha lateral, diâmetro sugeridos para linha lateral e pressão no início da linha lateral.

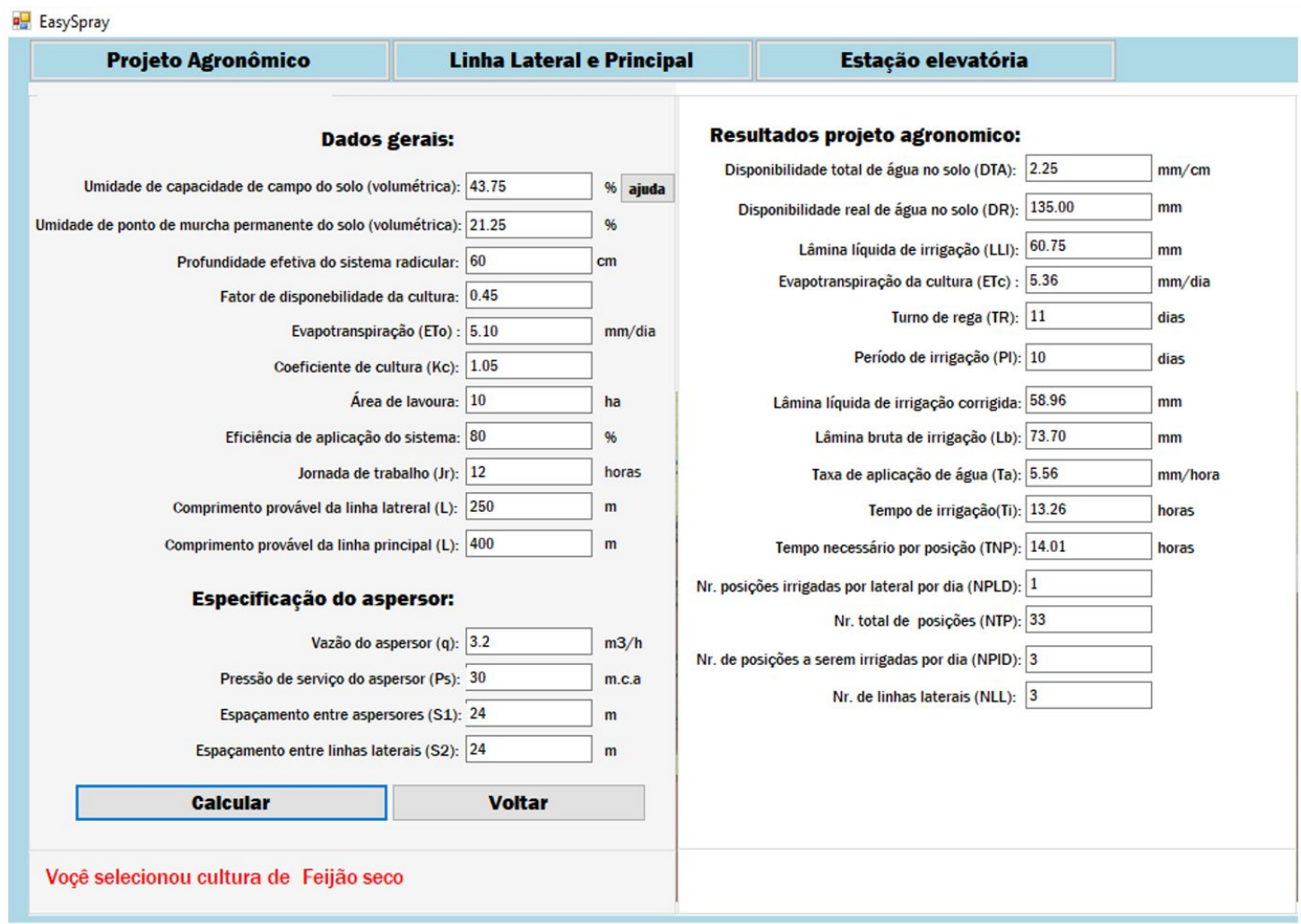

Figura 2 - Interface gráfica do software depois de se efetuar o uso de comando "Projeto agronômico"

Para dimensionamento da linha principal, é apresentada caixa de entrada em que é solicitado que o usuário selecione o leiaute da linha principal. Após, de maneira automatizada, são realizadas considerações acerca do número de linhas laterais para estimar o número de trechos da linha principal. Com isso, são sugeridos os diâmetros mínimos, fazendo-se necessária a entrada dos seguintes dados: distância desde a saída da bomba até o início da área, diâmetro interno da linha lateral, cotas no início e no final da linha principal. Em seguida, o usuário deverá clicar no comando "calcular", para que os cálculos sejam realizados e os resultados referentes a vazão da linha principal, perda de carga e o desnível, apresentados. Um detalhe importante nessa aba é que o software apresenta um desenho das linhas laterais e principal com os aspersores, a fim de facilitar a compressão do usurário

Em relação ao dimensionamento da linha lateral de aspersão convencional, quando comparado este software com outros, tais como o SLIDE (Andrini et al., 2019) e o IRRIGAR (Geisenhoff et al., 2018), nota-se que o Easyspray traz facilidades ao usuário no momento da aquisição de dados necessários para a realização dos cálculos necessários, por possuir biblioteca interna com informações referentes ao coeficiente de atrito "C" da equação de Hazen-Williams, que assume diferentes valores em função do material de fabricação da tubulação. Porém, há uma limitação do Easyspray por apresentar como opção única a adoção da equação de Hazen-Williams para estimativa da perda de carga que, segundo Carvalho (2014), não é recomendável para tubos com diâmetros inferiores a $50 \mathrm{~mm}$. Já os softwares desenvolvidos por Andrini et al. (2019) e Geisenhoff et al. (2018), utilizam equação de Darcy-Weisbach na estimativa das perdas de carga, aplicável para quaisquer condições de variáveis hidráulicas relativas ao escoamento de fluidos (Carvalho, 2014).

O software SLIDE também estima o comprimento máximo da linha lateral a fim de se ter a manutenção de uma determinada variação máxima de pressão na tubulação, além de realizar o 
dimensionamento da linha lateral trecho a trecho, fato que, de acordo com autores como Lopes et al. (2017) e Miranda et al. (2018), é o mais recomendável, por diminuir o risco de ocorrência de erros devido o emprego de coeficientes que corrigem a perda de carga em função do emprego de tubulações com múltiplas saídas. Já o software IRRIGAR (Geisenhoff et al., 2018) apresenta mais vantagens em relação ao Easyspray, no que se refere ao dimensionamento da linha lateral e principal pois, além de calcular os diâmetros dos tubos, este também estima os custos necessários para a sua aquisição.

No dimensionamento da linha principal, tanto o Easyspray como o IRRIGAR (Geisenhoff et al., 2018) efetuam os cálculos pelo método trecho a trecho utilizando equação de Hazen-Williams. Na Figura 3, pode-se ver a interface gráfica do software EASYSPRAY com a seleção do comando "Linha lateral e principal", com os seus respetivos resultados.

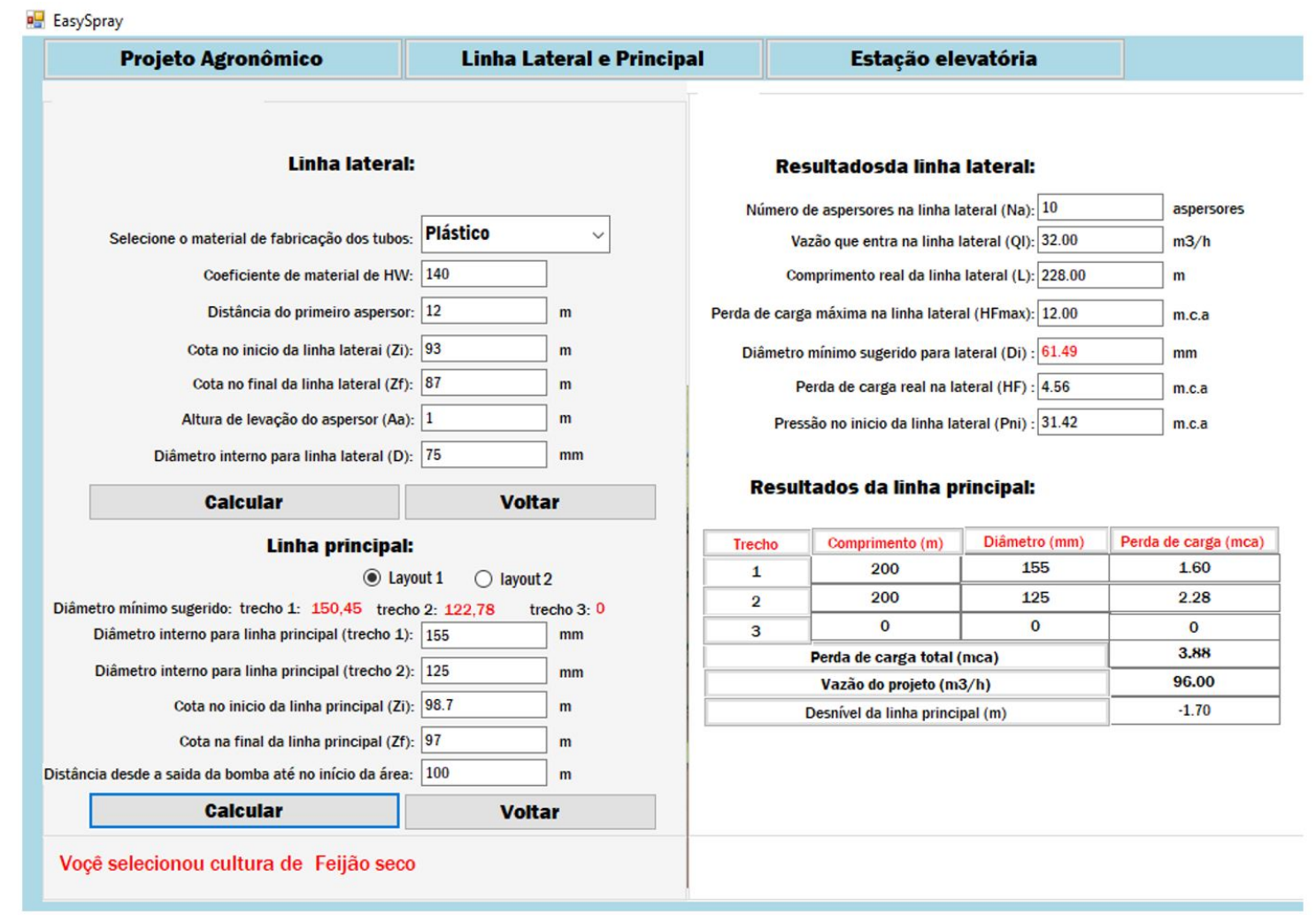

Figura 3 - Interface gráfica do software depois de se fazer o uso do comando "Linha lateral e principal"

O comando "Estação elevatória" leva o usuário a abrir a aba que lhe permite efetuar o dimensionamento da linha de sucção, adutora e motobomba. Com este comando, deve-se entrar com os seguintes dados: comprimento da linha de recalque, cotas no início e no final da linha de recalque e diâmetro para linha de recalque. No que se refere ao diâmetro da linha de recalque, o software já sugere um diâmetro mínimo que o usuário pode utilizar na escolha do diâmetro comercial.

Já para a linha de sucção é solicitado que usuário informe o comprimento da linha de sucção, coeficiente de Hazen-Williams, altura geométrica de sucção e diâmetro do tubo, também sendo sugerido um diâmetro mínimo, que o usuário pode se basear na escolha do tubo de sucção. A sugestão considera velocidade média máxima de escoamento de 1,5 m/s.

0 dimensionamento e seleção da motobomba requer as seguintes informações: rendimento da bomba e do motor. Há opção para o usuário escolher bomba com acionamento por motor Diesel ou elétrico. No caso em que se pretende utilizar bomba elétrica, o usuário deverá selecionar a opção "elétrica", e uma imagem de motobomba deste tipo é apresentada na interface gráfica do software. Já no caso que se pretende utilizar motor a Diesel, deverá ser selecionada a opção "Diesel”, e uma imagem de conjunto motobomba com acionamento a combustão de óleo Diesel é mostrada.

Nesta janela, ao ser indicado o comando "calcular", são apresentados os resultados referentes a desnível da linha de recalque, perda de carga na linha de recalque, perda de carga na sucção, potência da motobomba, altura manométrica da bomba, energia consumia por dia (para o caso de motobomba elétrica), consumo específico e consumo de óleo Diesel (caso de motobomba à Diesel). 
Comparando-se os procedimentos disponíveis para o dimensionamento da estação elevatória do Easyspray com outros aplicativos computacionais, nota-se que este possibilita a opção pelo uso de bombas acionadas por motores em que o combustível é o óleo Diesel, apresentando a quantidade de combustível necessária, o que permite a sua aplicabilidade em regiões sem a disponibilidade de energia elétrica transmitida de forma convencional. Adicionalmente, o Easyspray apresenta imagens ilustrativas na sua interface gráfica, o que pode facilitar a interpretação dos resultados, principalmente para casos em que os usuários são técnicos sem qualificação avançada específica na área de irrigação, como é o caso de muitos técnicos moçambicanos. Já o software IRRIGAR (Geisenhoff et al., 2018), permite a estimativa dos custos para aquisição da tubulação de sução e recalque e da motobomba, mas sua interface gráfica é complexa, o que pode dificultar seu uso. Em relação à apresentação de custos, o Easyspray não adota este procedimento em função da possível variação decorrente de flutuações cambiais e inflacionárias.

São apresentadas nas Figuras 4 e 5 as interfaces gráficas do software Easyspray com os resultados, considerando os casos de motobomba elétrica e à Diesel, respetivamente.

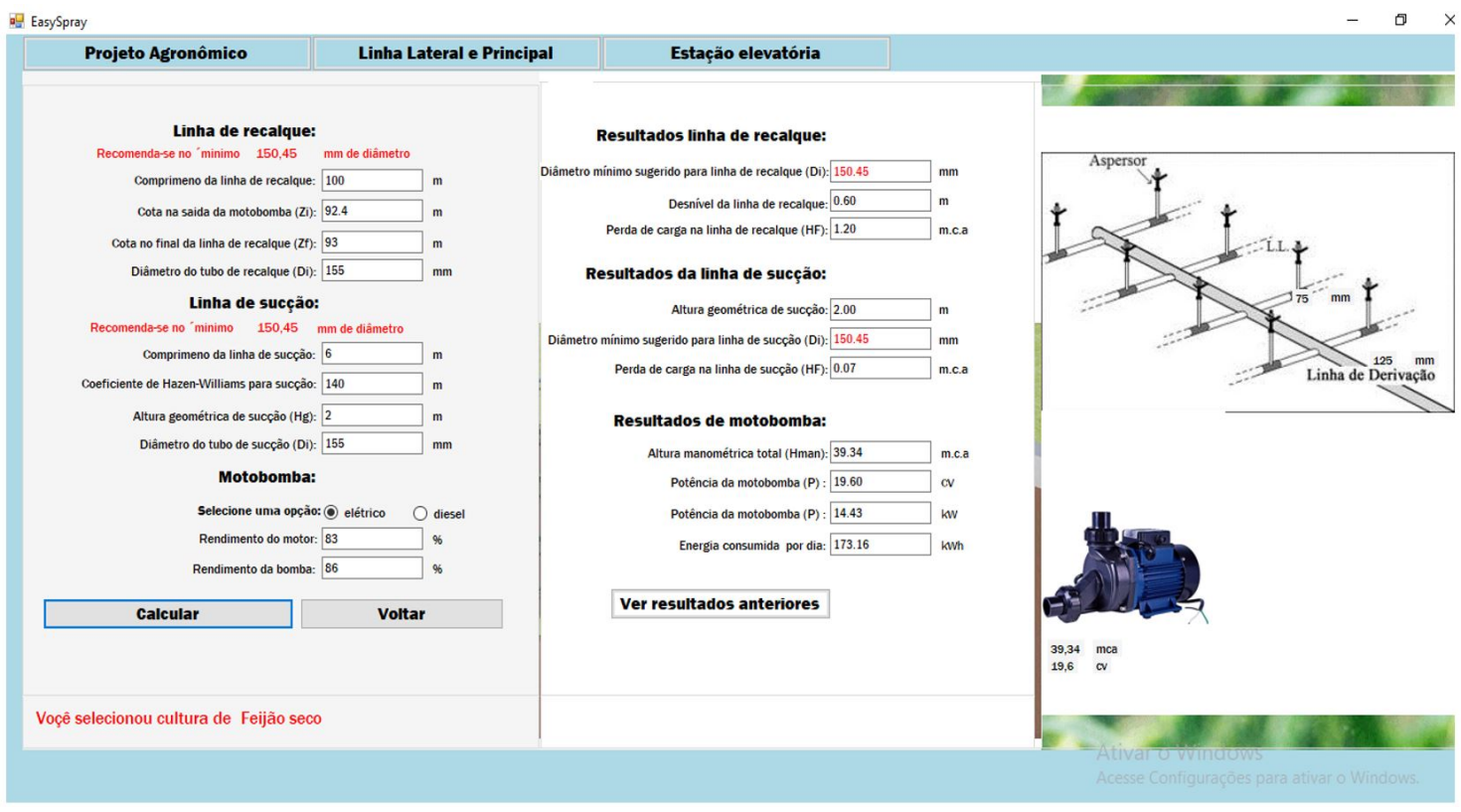

Figura 4 - Interface gráfica do software com os resultados, considerando motobomba elétrica

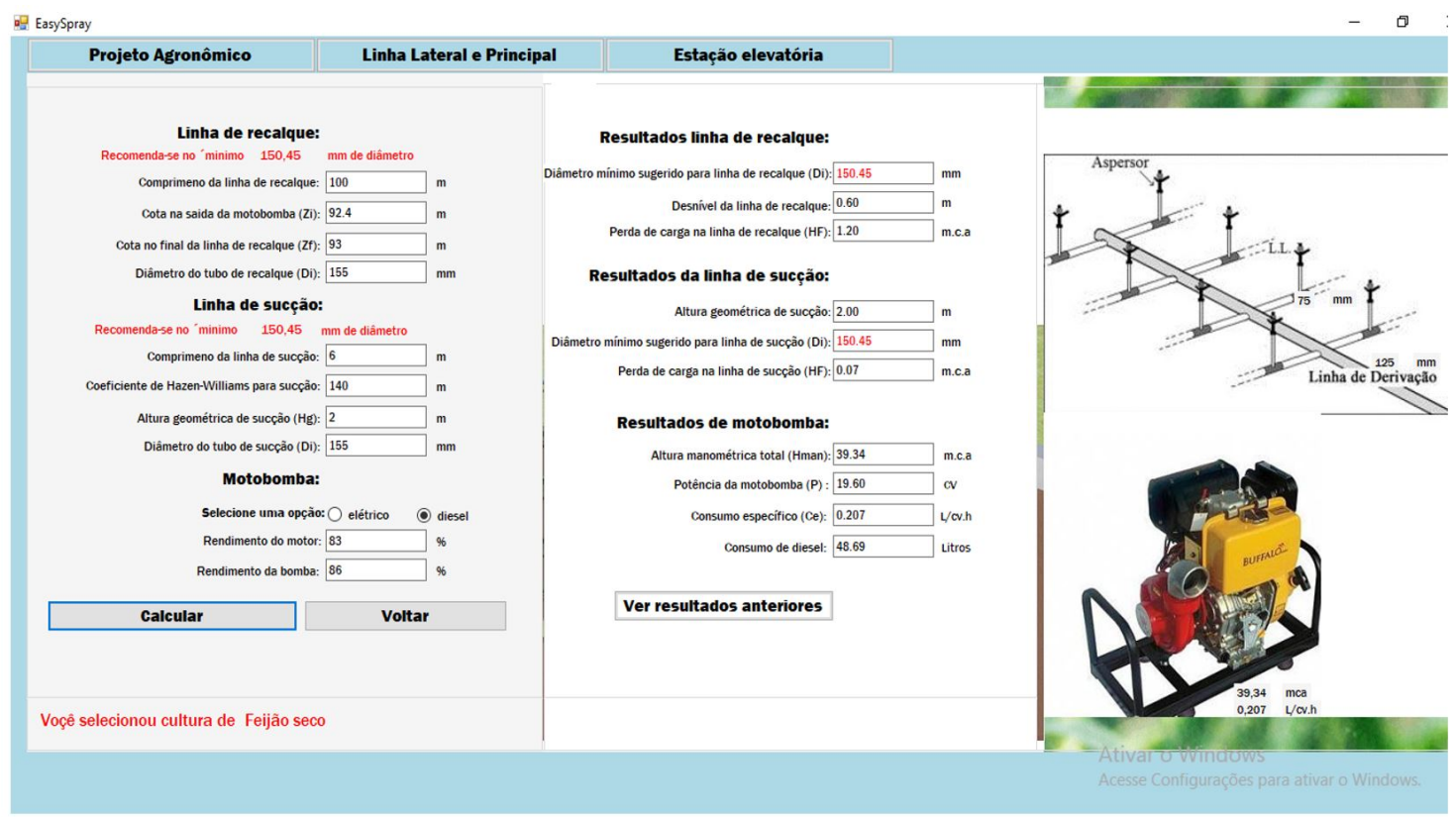

Figura 5 - Interface gráfica do software com os resultados, para motobomba à óleo Diesel 
Quando foi testado o software para dimensionamento de um sistema de irrigação por aspersão convencional em 10 ha de cultivo de feijão por uma associação de produtores rurais em Moçambique, no distrito de Chimoio, os resultados obtidos no cálculo por meio do software mostraram-se semelhantes aos do dimensionamento manual. Para aba do projeto agronômico (Tabela 1), quase todos os resultados obtidos com o software foram iguais ao resultados de cálculo manual, exceto no que se refere ao tempo de irrigação e tempo necessário por posição na linha lateral, que apresentaram uma ligeira diferença nas casas decimais quando comparados com o cálculo manual devida provavelmente a arredondamentos.

Tabela 1 - Resultados referentes ao projeto agronômico obtidos por meio de cálculo manual e cálculo com software

\begin{tabular}{l|c|c}
\hline \multicolumn{1}{c}{ Itens calculados } & Manual & Software \\
\hline Disponibilidade real de água no solo (mm) & 135,00 & 135,00 \\
\hline Lâmina líquida de irrigação (mm) & 60,75 & 60,75 \\
\hline Evapotranspiração da cultura (mm dia-1) & 5,36 & 5,36 \\
\hline Turno de rega (dia) & 11 & 11 \\
\hline Lâmina líquida de irrigação corrigida (mm) & 58,91 & 58,96 \\
\hline Lâmina bruta de irrigação (mm) & 73,63 & 73,70 \\
\hline Taxa de aplicação de água (mm h-1) & 5,56 & 5,56 \\
\hline Tempo de irrigação (h) & 13,25 & 13,26 \\
\hline Tempo necessário por posição na linha lateral (h) & 13,75 & 14,01 \\
\hline Número de posições irrigadas por lateral por dia & 1 & 1 \\
\hline Número total de posições & 33 & 33 \\
\hline Número de posições a serem irrigadas por dia & 3 & 3 \\
\hline Número de linhas laterais & 3 & 3 \\
\hline
\end{tabular}

No dimensionamento da linha lateral (Tabela 2), a maioria dos resultados obtidos com o software foram iguais aos obtidos por cálculo manual, exceto os referentes à perda de carga real na linha lateral e pressão na entrada da linha lateral, também em função de arredondamentos. Já para linha principal e linha de recalque (Tabela 3), a diferença foi somente nos resultados referentes a perda de carga da linha principal e perda de carga na linha de recalque, pelo mesmo motivo supracitado. Para linha principal e motobomba (Tabela 4), notou-se uma ligeira diferença nos resultados referentes à altura manométrica (em caso de bomba elétrica), potência da motobomba e volume de Diesel consumido (em caso de motobomba a Diesel), porém, essas diferenças não possuem impacto significativo no sistema de irrigação. O software, então, mostrou ser ferramenta interessante quanto à rapidez na efetuação dos cálculos e prestar-se para análise rápida de diferentes cenários passíveis de instalação.

Tabela 2 - Resultados referentes ao dimensionamento da linha lateral obtidos por meio de cálculo manual e cálculo com software

\begin{tabular}{l|c|c}
\hline \multicolumn{1}{c}{ Linha lateral } \\
\multicolumn{1}{c}{ Itens calculados } & Manual & Software \\
\hline Número de aspersores na linha lateral & 10 & 10 \\
\hline Vazão da linha lateral & 32 & 32 \\
\hline Comprimento real da linha lateral (m) & 228 & 228 \\
\hline Perda de carga máxima na linha lateral mca) & 12 & 12 \\
\hline Diâmetro de linha lateral (mm) & 75 & 75 \\
\hline Perda de carga real na linha lateral (mca) & 4,44 & 4,56 \\
\hline Pressão na entrada da linha lateral (mca) & 31,33 & 31,42 \\
\hline
\end{tabular}


Tabela 3 - Resultados referentes ao dimensionamento da linha principal e recalque obtidos por meio de cálculo manual e cálculo com software

\begin{tabular}{l|c|c}
\hline \multicolumn{1}{c}{ Linha principal e recalque } \\
\hline \multicolumn{1}{c}{ Itens calculados } & Manual & Software \\
\hline Vazão na linha principal, trecho $1\left(\mathrm{~m}^{3} \mathrm{~h}^{-1}\right)$ & 96 & 96 \\
\hline Vazão na linha principal, trecho $2\left(\mathrm{~m}^{3} \mathrm{~h}^{-1}\right)$ & 64 & 64 \\
\hline Diâmetro da linha principal, trecho 1 (mm) & 155 & 155 \\
\hline Diâmetro da linha principal, trecho 2 (mm) & 125 & 125 \\
\hline Perda de carga na linha principal (m) & 4,90 & 4,81 \\
\hline Comprimento da linha de recalque (m) & 100 & 100 \\
\hline Diâmetro da linha de recalque (mm) & 155 & 155 \\
\hline Perda de carga na linha de recalque (mca) & 1,44 & 1,20 \\
\hline
\end{tabular}

Tabela 4 - Resultados referentes ao dimensionamento da linha de sucção e motobomba obtidos por meio de cálculo manual e cálculo com software

\begin{tabular}{l|c|c}
\hline \multicolumn{1}{c}{ Itens calculados } & Manual & Software \\
\hline Altura geométrica de sucção (m) & 2 & 2 \\
\hline Comprimento da sucção (m) & 6 & 6 \\
\hline Diâmetro de linha de sucção (mm) & 155 & 155 \\
\hline Perda de carga na linha de sucção (mca) & 0,07 & 0,07 \\
\hline Altura manométrica total da motobomba (m) & 41,30 & 40,32 \\
\hline Potência da motobomba (c.v.) & 18,36 & 17,92 \\
\hline Energia consumida por dia (kWh) & 161,93 & 158,28 \\
\hline Consumo específico do motor (L cv-1 ${ }^{-1}$ ) & 0,21 & 0,21 \\
\hline Volume de Diesel consumido (L) & 46,14 & 45,62 \\
\hline
\end{tabular}

\section{CONCLUSÕES}

O software é uma ferramenta fácil de utilizar, quando comparado com outros aplicativos disponíveis para dimensionamento de projetos de irrigação por aspersão convencional, mostrando, como principais vantagens em relação a outras aplicações computacionais, a disponibilidade de biblioteca interna com os principais dados a serem utilizados para dimensionamento do sistema de irrigação e considerar também a possibilidade de bombeamento a partir do uso de bombas acionadas por motores Diesel, que podem ser aplicadas em localidades sem disponibilidade de energia elétrica.

Pode ser instalado em todos os computadores que possuam Windows, sem depender de programas auxiliares e a sua interface gráfica é intuitiva para o usuário que tenha um treinamento mínimo em Engenharia de Irrigação. Assim, o software mostrou ser eficiente e eficaz em efetuar cálculos e dimensionamentos, quando comparado ao trabalho realizado manualmente.

O software será disponibilizado gratuitamente no sítio eletrônico do Programa de PósGraduação em Recursos Hídricos da Universidade Federal de Lavras (Universidade Federal de Lavras, 2020)

\section{REFERÊNCIAS}

Andrini, M., Souza, C. A. N., \& Saad, J. C. C. (2019). Software SLIDE para dimensionamento de linhas laterais em irrigação por aspersão convencional. Irriga, 1(1), 18-24.

Azevedo Neto, J. M. Manual de hidráulica (cap. 11, pp. 255- 293). São Paulo: Blucher 2015.

Bernardo, S., Soares, A. A., \& Mantovani, E. C. (2008). Manual de irrigação (cap. 8, pp. 407-431). Viçosa. 
Bertossi, A. P., Milen, L. C., \& Hott, M. O., Rodrigues, R. R., \& Reis, E. F. (2013). Avaliação de um sistema de irrigação por aspersão em malha em pastagem. Nucleus, 10(1), 24-36.

Campêlo, A. R., Fernandes, C. N. V., Silva, A. R. A., Oliveira, M. R. M., Bezerra, F. M. L., \& Cândido, M. J. D. (2014) Avaliação de sistemas de irrigação por aspersão em malha em áreas cultivadas com capim-braquiária. Agropecuária Técnica, 1(35), 1-12.

Carvalho, D. F. (2014). Instalações de recalque e bombeamento para sistemas de irrigação (cap. 25, pp. 347-367). Lavras: Editora UFLA.

Carvalho, D. F., Oliveira, L. F. C. (2012). Planejamento e manejo de água na agricultura irrigada (cap. 1, pp. 1164). Viçosa: UFV.

Food and Agriculture Organization of the United Nations - FAO. (1990). Irrigation and Drainage Paper 56. Crop Evapotranspiration. Roma: FAO. Recuperado em 14 de abril de 2020, de http://www.fao.org/landwater/databases-and-software/climwat-for-cropwat/en/

Food and Agriculture Organization of the United Nations - FAO. (2006). Climwat 2.0. Roma: FAO. Recuperado em 14 de abril de 2020, de http://www.fao.org/land-water/databases-and-software/climwat-forcropwat/en/

Frigo, J. P., Vilas Boas, M. A., Frigo, J. P., \& Frigo, E. P. (2016). comparação entre gráficos de controle de shewhart, cusum e mmep no processo de irrigação por aspersão convencional. Irriga, 1(1), 56-70.

Geisenhoff, L. O., Flores, D. M., Gomes Filho, R. R., Santos, R. C., Jordan, R. A., \& Oliveira, L. C. (2018). Irrigar aplicativo computacional para o dimensionamento econômico de sistemas de irrigação por aspersão convencional. Revista Brasileira de Agricultura Irrigada, 12(4), 2761-2775.

Geisenhoff, L. O., Oliveira, F. C., Biscaro, G. A., Almeida, A. S., \& Schwerz, F. (2015). Produtividade do brócolis-decabeça sob diferentes sistemas de irrigação. Engenharia Agrícola, 35(5), 863-874.

Locatelli, V. E. R., Medeiros, R. D., Smiderle, O. J., Albuquerque, J. A. A., Wellington, F., Araújo, W. F., \& Souza, K. T. S. (2014). Componentes de produção, produtividade e eficiência da irrigação do feijão-caupi no cerrado de Roraima. Revista Brasileira de Engenharia Agrícola e Ambiental, 18(6), 574-580.

Lopes, J. D., Lima, F. Z., \& Oliveira, F. G. (2017). Irrigação por aspersção convencional (cap. 6, p. 300-340). Viçosa, MG.

Mantovani, E. C., Bernardo, S., \& Palaretti, L. F. (2009). Irrigação princípios e métodos (cap. 3, pp. 111-215). Viçosa, MG.

Miranda, E. P., Rosal, G. B., \& Lima, F. U. (2018). Diferenças entre o método trecho-a-trecho e o método de múltiplas saídas na determinação das perdas de cargas em uma linha lateral de um sistema de irrigação localizada. Revista Brasileira de Gestão Ambiental e Sustentabilidade, 5(11), 911-918.

Moçambique. Ministério da Agricultura de Moçambique - MASA. (2017). Estratégia de irrigação em Moçambique (54 p.). Maputo. Recuperado em 14 de abril de 2020, de http://www.inir.gov.mz/files/201407/Estrategia\%20de\%20Irrigacao.pdf

Pereira, L. R., Souza, J. M., \& Rafael, A. M., Cruz, E. A., Teixeira, A. G., \& Reis, E. F. (2016). Uniformidade e eficiência de aplicação de água em um sistema de irrigação por aspersão em pastagem. Agrarian (Dourados), 9(32), 156-161.

Perroni, B. L. T., Faria, L. C., Carvalho, J. A., \& Oliveira, H. F. E. (2015). Influência do custo da energia elétrica e do material da tubulação na velocidade econômica de bombeamentos. Irriga, 20(1), 13-20.

Pinto, U. R. C., Ribeiro, P. H. P., Salomão, L. C., Cantuario, F. S., Brito, R. R., Silva, T. T. S., \& Coneglian, A. (2015). Uniformidade de distribuição de água em aspersão convencional sob diferentes pressões de serviços. Global. Science and Technology, 8(2), 160-169.

Rodrigues, L. G., Nery, A. R., Sousa, F. R. R., \& Rodrigues, L. N. (2019). Coeficientes de uniformidade em aspersores de baixa vazão com diferentes arranjos e altura da haste. Revista Verde de Agroecologia e Desenvolvimento Sustentável, 14(2), 170-180.

Rodrigues, R. R., Cola, M. P. A., Nazário, A. A., De Azevedo, J. M. G., \& Reis, E. F. (2013). Eficiência e uniformidade de um sistema de irrigação por gotejamento na cultura do cafeeiro. Ambiência - Revista do Setor de Ciências Agrárias e Ambientais, 9(2), 323-334.

Silva, G. J., \& Klar, A. E. (2010). Modelo de software livre para avaliação de sistema de irrigação por pivô central. Irriga, 25(1), 70-84.

Universidade Federal de Lavras - UFLA, \& Programa de Pós-graduação em Recursos Hídricos. (2020). Lavras: UFLA. Recuperado em 14 de abril de 2020, de https://sigaa.ufla.br/sigaa/public/programa/portal.jsf?lc=pt_BR\&id=1787 


\section{Contribuições dos autores}

Fidel Luís Rodrigues Tambo. Participou na obtenção de Dados de Moçambique, criação de interface gráfica do software, elaboração dos códigos de programação e criação das rotinas computacionais;

Michael Silveira Thebaldi, Participou na revisão das equações, contribuição; correção das equações do Software, contribuição com ideias para melhorar a interface gráfica do software a fim de torná-lo mais simples para o usuario;

Luiz Antonio Lima,. Participou na correção e Revisão do software, correção contribuição com ideias para melhorar a interface gráfica do software a fim de torná-lo mais simples para o usuario. 